IJMMS 31:9 (2002) 555-565

PII. S0161171202012553

http://ijmms.hindawi.com

(c) Hindawi Publishing Corp.

\title{
GENERALIZED HAMILTONIAN BIODYNAMICS AND TOPOLOGY INVARIANTS OF HUMANOID ROBOTS
}

\author{
VLADIMIR IVANCEVIC
}

Received 28 March 2001 and in revised form 9 January 2002

\begin{abstract}
Humanoid robots are anthropomorphic mechanisms with biodynamics that resembles human musculo-skeletal dynamics. This paper proposes a new generalized (dissipative, muscle-driven, stochastic) Hamiltonian model of humanoid biodynamics. Also, (co)homological analysis is performed on its Lie-group based configuration and momentum phasespace manifolds.
\end{abstract}

2000 Mathematics Subject Classification: 37J05, 68T40.

1. Introduction. Humanoid robots are human-like, anthropomorphic mechanisms with complex, muscle driven, biodynamics with many degrees-of-freedom [8]. This paper proposes a new generalized Hamiltonian model of humanoid biodynamics, together with topology analysis of its configuration and momentum phase-space manifolds.

Since the early work of Vukobratovic on exoskeletal robotic-suite (see [18, 19]), the vast body of research has been done in relation to kinematics, dynamics, and control of biped, anthropomorphic and humanoid robots [6, 13, 17, 19]. Some of the biped models had the ability of passive dynamic walking and others had powered walking ability (see [19]). The previous decade was dominated by various solutions to the kinematic problems of redundancy and singularities [20]. The last decade has been characterized mostly by extensive use of intelligent, neuro-fuzzy genetic control of humanoid dynamics [3, 12, 14, 15], and computer-graphics animation [9].

In this paper, the $N$-dimensional configuration manifold $Q^{N}$ of humanoid biodynamics (HB, for short) is constructed using direct products of constrained rotational Lie groups $\mathrm{SO}(n)$ (for $n=2,3$ ). A two-stage, generalized Hamiltonian formulation of HB is performed on the momentum phase-space manifold (i.e., cotangent bundle) $T^{*} Q^{N}$, using symplectic geometry of rotational Lie groups $\mathrm{SO}(n)$ and their algebras so $(n)$. A dissipative, muscle driven, Hamiltonian HB is first deterministically formulated on $T^{*} Q^{N}$, and afterwards stochastically generalized to include noise in the form of both diffusion fluctuations and discontinuous master jumps. Topological analysis of $T^{*} Q^{N}$ is performed using both cohomology and homology groups. In the case of reduced configuration manifold, the Betti numbers and Euler-Poincaré characteristic are given.

\section{Generalized Hamiltonian HB}

2.1. Configuration manifold of HB. Kinematics of an $n$-segment humanoid chain is usually defined as a map between external (usually, end-effector) coordinates 
$x^{r}(r=1, \ldots, n)$ and internal (joint) coordinates $q^{i}(i=1, \ldots, N)[7,8]$. The forward kinematics are defined as a nonlinear map $x^{r}=x^{r}\left(q^{i}\right)$ with a corresponding linear vector functions $d x^{r}=\partial x^{r} / \partial q^{i} \cdot d q^{i}$ of differentials and $\dot{x}^{r}=\partial x^{r} / \partial q^{i} \cdot \dot{q}^{i}$ of velocities. (Here and subsequently the summation convention over repeated indices is understood.) When the rank of the configuration-dependent Jacobian matrix $J \equiv$ $\partial x^{r} / \partial q^{i}$ is less than $n$ the kinematic singularities occur; the onset of this condition could be detected by the manipulability measure. Inverse kinematics are defined conversely by a nonlinear map $q^{i}=q^{i}\left(x^{r}\right)$ with a corresponding linear vector functions $d q^{i}=\partial q^{i} / \partial x^{r} \cdot d x^{r}$ of differentials and $\dot{q}^{i}=\partial q^{i} / \partial x^{r} \cdot \dot{x}^{r}$ of velocities. As HB is highly redundant $(n<N)$, the inverse kinematic problem admits infinite solutions; therefore, the pseudo-inverse configuration-control is used instead: $\dot{q}^{i}=J^{*} \dot{x}^{r}$, where $J^{*}=J^{T}\left(J J^{T}\right)^{-1}$ denotes the Moore-Penrose pseudo-inverse of the Jacobian matrix $J$.

Humanoid joints, that is, internal coordinates $q^{i}(i=1, \ldots, N)$, constitute a smooth configuration manifold $Q^{N}$, described as follows. Uniaxial, hinge joints represent constrained, rotational Lie groups $\mathrm{SO}(2)_{\text {cnstr }}^{i}$, parameterized by constrained angles $q_{\text {cnstr }}^{i} \equiv q^{i} \in\left[q_{\min }^{i}, q_{\text {max }}^{i}\right]$. Three-axial, ball-and-socket joints represent constrained rotational Lie groups $\mathrm{SO}(3)_{\mathrm{cnstr}}^{i}$, parameterized by constrained Euler angles $q^{i}=q_{\text {cnstr }}^{\phi_{i}}$ (in the following text, the subscript cnstr will be omitted, for the sake of simplicity, and always assumed in relation to internal coordinates $\left.q^{i}\right)$. All SO $(n)$-joints are Hausdorff $C^{\infty}$-manifolds with atlases $\left(U_{\alpha}, u_{\alpha}\right)$; in other words, they are paracompact and metrizable smooth manifolds, admitting Riemannian metric.

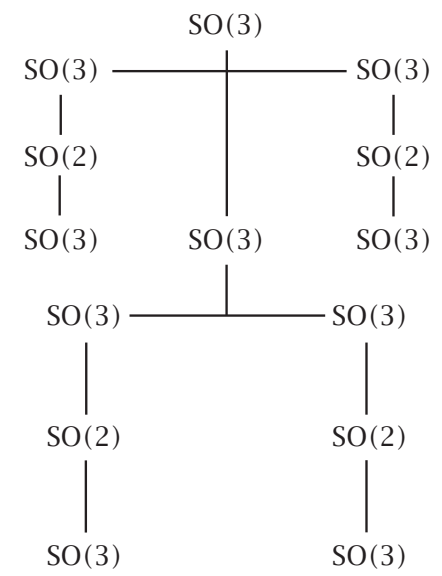

FIGURE 2.1. Humanoid configuration manifold $Q^{N}$ modeled as anthropomorphic product tree of constrained rotational $\mathrm{SO}(n)$ groups (for $n=2,3$ ).

Let $A$ and $B$ be two smooth manifolds described by smooth atlases $\left(U_{\alpha}, u_{\alpha}\right)$ and $\left(V_{\beta}, v_{\beta}\right)$, respectively. Then, the family $\left(U_{\alpha} \times V_{\beta}, u_{\alpha} \times v_{\beta}: U_{\alpha} \times V_{\beta} \rightarrow \mathbb{R}^{m} \times \mathbb{R}^{n}\right)_{(\alpha, \beta)} \in$ $A \times B$ is a smooth atlas for the direct product $A \times B$. Now, if $A$ and $B$ are two Lie groups (say, $\mathrm{SO}(n)$, for $n=2,3$ and $i=1, \ldots, N$ ), then their direct product $G=A \times B$ is at the same time their direct product as smooth manifolds and their direct product as 
algebraic groups, with the product law

$$
\left(a_{1}, b_{1}\right)\left(a_{2}, b_{2}\right)=\left(a_{1} a_{2}, b_{1} b_{2}\right), \quad a_{1,2} \in A, b_{1,2} \in B .
$$

Generalizing the direct product to $N$ rotational joint groups, we can draw an anthropomorphic product tree (see Figure 2.1) using a line segment “-” to represent direct products of humanoid's $\mathrm{SO}(n)$-joints. This is our basic model of the humanoid configuration manifold $Q^{N}$.

Now, on the HB configuration manifold $Q^{N}$, we are going to perform some categorical transformations (see [10], as well as $[7,8]$ ). If we apply the functor Lie to the category ${ }^{\bullet}\left[\mathrm{SO}(n)^{i}\right]$ of rotational Lie groups $\mathrm{SO}(n)^{i}$ and their homomorphisms we obtain the category $\cdot\left[\operatorname{so}(n)_{i}\right]$ of corresponding tangent Lie algebras so $(n)_{i}$ and their homomorphisms. If we further apply the isomorphic functor Dual to the category . $\left[\operatorname{so}(n)_{i}\right]$, we obtain the dual category $*^{*}\left[\operatorname{so}(n)_{i}^{*}\right]$ of cotangent, or, canonical Lie algebras so $(n)_{i}^{*}$ and their homomorphisms. To go directly from ${ }^{\bullet}\left[\mathrm{SO}(n)^{i}\right]$ to ${ }^{*}\left[\operatorname{so}(n)_{i}^{*}\right]$, we use the canonical functor Can $[7,8]$. Therefore, we have a commutative triangle

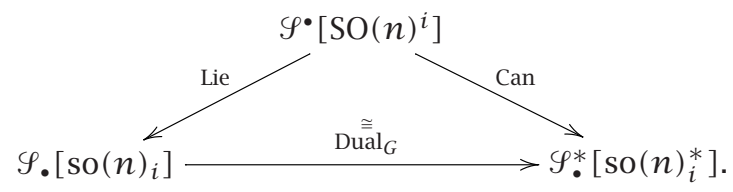

Both the tangent algebras so $(n)_{i}$ and the cotangent algebras so $(n)_{i}^{*}$ contain infinitesimal group generators, angular velocities $\dot{q}^{i}=\dot{q}^{\phi_{i}}$ in the first case and canonical angular momenta $p_{i}=p_{\phi_{i}}$ in the second [15]. As Lie group generators, angular velocities and angular momenta satisfy the respective commutation relations $\left[\dot{q}^{\phi_{i}}, \dot{q}^{\psi_{i}}\right]=$ $\epsilon_{\theta}^{\phi \psi} \dot{q}^{\theta_{i}}$ and $\left[p_{\phi_{i}}, p_{\psi_{i}}\right]=\epsilon_{\phi \psi}^{\theta} p_{\theta_{i}}$, where the structure constants $\epsilon_{\theta}^{\phi \psi}$ and $\epsilon_{\phi \psi}^{\theta}$ constitute totally antisymmetric third-order tensors.

In this way, the functor $\operatorname{Dual}_{\mathrm{G}}: \mathrm{Lie} \cong$ Can establishes a geometrical duality between kinematics of angular velocities $\dot{q}^{i}$ (involved in Lagrangian formalism on the tangent bundle of $Q^{N}$ ) and that of angular momenta $p_{i}$ (involved in Hamiltonian formalism on the cotangent bundle of $Q^{N}$ ). This is analyzed below. In other words, we have two functors Lie and Can from a category of Lie groups (of which $\mathscr{S}^{\bullet}\left[\mathrm{SO}(n)^{i}\right]$ is a subcategory) into a category of their Lie algebras (of which $\mathscr{S}_{\bullet}\left[\operatorname{so}(n)_{i}\right]$ and $\mathscr{S}_{\bullet}^{*}\left[\operatorname{so}(n)_{i}^{*}\right]$ are subcategories), and a natural equivalence (functor isomorphism) between them defined by the functor Dual ${ }_{G}$. (As angular momenta $p_{i}$ are in a bijective correspondence with angular velocities $\dot{q}^{i}$, every component of the functor Dual $\mathrm{G}_{\mathrm{G}}$ is invertible.)

Applying the functor Lie to the HB-configuration manifold $Q^{N}$ (Figure 2.1), we get the product tree of the same anthropomorphic structure, but having tangent Lie algebras $\mathrm{SO}(n)_{i}$ as vertices, instead of the groups $\mathrm{SO}(n)^{i}$. Again, applying the functor Can to $Q^{N}$, we get the product tree of the same anthropomorphic structure, but this time having cotangent Lie algebras so $(n)_{i}^{*}$ as vertices. Both the tangent algebras so $(n)_{i}$ and the cotangent algebras so $(n)_{i}^{*}$ contain infinitesimal group generators: angular velocities $\dot{q}^{i}=\dot{q}^{\phi_{i}}$-in the first case, and canonical angular momenta $p_{i}=p_{\phi_{i}}$-in the second case $[7,8]$. As Lie group generators, both the angular velocities and the angular momenta satisfy the commutation relations: $\left[\dot{q}^{\phi_{i}}, \dot{q}^{\psi_{i}}\right]=\epsilon_{\theta}^{\phi \psi} \dot{q}^{\theta_{i}}$ and $\left[p_{\phi_{i}}, p_{\psi_{i}}\right]=\epsilon_{\phi \psi}^{\theta} p_{\theta_{i}}$, 
respectively, where the structure constants $\epsilon_{\theta}^{\phi \psi}$ and $\epsilon_{\phi \psi}^{\theta}$ constitute the totally antisymmetric third-order tensors.

The functor Lie defines the second-order Lagrangian formulation on the tangent bundle $T Q^{N}$ (i.e., the velocity phase-space manifold) while the functor Can defines the first order canonical Hamiltonian formalism on the cotangent bundle $T^{*} Q^{N}$ (i.e., the momentum phase-space manifold). As these two formalisms are related by the isomorphic functor Dual, they are equivalent. Now, as it is easier to make a stochastic generalization on the first order equations, we will follow the canonical Hamiltonian functor Can.

2.2. Dissipative, muscle-driven, Hamiltonian HB. The Hamiltonian function $H$ : $T^{*} Q^{N} \rightarrow \mathbb{R}$ of HB is, in local canonical coordinates $q^{i}, p_{i} \in U_{p}$ on the momentum phase-space manifold $T^{*} Q^{N}$, given by the equation

$$
H(q, p)=\frac{1}{2} g^{i j}(q, m) p_{i} p_{j}+V(q),
$$

where $g^{i j}(q, m)$ denotes the contravariant material metric tensor, relating internal and external coordinates, and including $n$ segmental masses $m_{\chi}$ as

$$
g^{i j}(q, m)=\sum_{x=1}^{n} m_{\chi} \delta_{r s} \frac{\partial q^{i}}{\partial x^{r}} \frac{\partial q^{j}}{\partial x^{s}} .
$$

Deterministic $\mathrm{HB}$, representing the canonical functor Can: $\left[\mathrm{SO}(n)^{i}\right] \Rightarrow{ }_{\bullet}^{*}\left[\operatorname{so}(n)_{i}^{*}\right]$, is given by dissipative, driven $\delta$-Hamiltonian equations,

$$
\begin{aligned}
\dot{q}^{i} & =\frac{\partial H}{\partial p_{i}}+\frac{\partial R}{\partial p_{i}}, \\
\dot{p}_{i} & =F_{i}-\frac{\partial H}{\partial q^{i}}+\frac{\partial R}{\partial q^{i}}, \\
q^{i}(0) & =q_{0}^{i}, \quad p_{i}(0)=p_{i}^{0},
\end{aligned}
$$

including contravariant equation (2.5) - the velocity vector-field, and covariant equation (2.6) - the force one-form, together with initial joint angles and momenta. Here $(i=1, \ldots, N)$, and $R=R(q, p)$ denotes the Raileigh nonlinear (biquadratic) dissipation function, and $F_{i}=F_{i}(t, q, p)$ are covariant driving torques of equivalent muscular actuators, resembling muscular excitation and contraction dynamics in rotational form $[7,8]$.

The velocity vector field (2.5) and the force one-form field (2.6) together define the generalized Hamiltonian vector field $X_{H}$, which geometrically represents the section of the momentum phase-space manifold $T^{*} Q^{N}$, which is itself the cotangent bundle of the HB-configuration manifold $Q^{N}$, (Figure 2.1); the Hamiltonian (total energy) function $H=H(q, p)$ is its generating function.

As an example, we have considered the common humanoid task of symmetrical $3 D$ load lifting $[7,8]$. The general HB-configuration manifold $Q^{N}$ (Figure 2.1) is now reduced to the 9-dimensional torus $T^{9}$ and the momentum phase-space manifold corresponds to its 18 -dimensional cotangent bundle $T^{*} T^{9}$. In this case, the canonical 
velocity and force equations (2.5) and (2.6) obtain relatively simple expanded form (where symbolically derived using computer algebra, [7, 8])

$$
\begin{aligned}
\dot{q}^{i}=p_{i} & \left\{\left[J_{i}\right]^{-1}+\left[m_{i}\left(\sum_{j=1}^{i} L_{j} \cos q^{j}\right)^{2}\right]^{-1}\right\}+\frac{\partial R}{\partial p_{i}}, \\
\dot{p}_{i}= & F_{i}\left(t, q^{i}, p_{i}\right)-g \sum_{j=i}^{10-i} L_{j} m_{j} \sin q^{j} \\
& -\sum_{j=i}^{10-i} L_{j} \sin q^{j} p_{i} p_{j}\left[m_{i}\left(\sum_{k=1}^{i} L_{k} \cos q^{k}\right)^{3}\right]^{-1}+\frac{\partial R}{\partial q^{i}},
\end{aligned}
$$

for $(i=1, \ldots, 9)$. Here $L s, m s$, and $J$ s denote the segment lengths, masses, and moments of inertia, respectively, and $g$ is the common gravity constant. In the contravariant-velocity equation (2.8) the terms denote rotational velocities, translational velocities and velocity-dumping for the $i$ th joint, respectively, whereas the covariantforce equation (2.9) contains terms of muscular excitation/contraction actuator torques $F_{i}=F_{i}\left(t, q^{i}, p_{i}\right)[7,8]$, and passive-external torques (gravitational, Coriolis, centrifugal, and joint-damping, respectively).

As a Lie group, the configuration manifold $Q^{N}$ is Hausdorff. Therefore, for $x=$ $\left(q^{i}, p_{i}\right) \in U_{p}, U_{p}$ open in $T^{*} Q^{N}$, there exists a unique one-parameter group of diffeomorphisms $\phi_{\delta_{t}}: T^{*} Q^{N} \rightarrow T^{*} Q^{N}$, the deterministic $\delta$-Hamiltonian phase-flow [7, 8]

$$
\begin{gathered}
\phi_{\delta_{t}}: G_{1} \times T^{*} Q^{N} \longrightarrow T^{*} Q^{N},(p(0), q(0)) \longmapsto(p(t), q(t)), \\
\left(\phi_{\delta_{t}} \circ \phi_{\delta_{s}}=\phi_{\delta_{t+s}}, \phi_{\delta_{0}}=\text { identity }\right),
\end{gathered}
$$

given by (2.5), (2.6), and (2.7) such that

$$
\left.\frac{d}{d t}\right|_{t=0} \phi_{\delta_{t}} x=J \nabla H(x) .
$$

2.3. Stochastic Hamiltonian HB. The general $N$-dimensional Markov process can be defined by Ito stochastic differential equation (summing upon repeated indexes assumed) [16]

$$
\begin{aligned}
d x_{i}(t) & =A_{i}\left[x_{i}(t), t\right] d t+B_{i j}\left[x_{i}(t), t\right] d W^{j}(t), \\
x_{i}(0) & =x_{i 0}, \quad(i, j=1, \ldots, N)
\end{aligned}
$$

or corresponding Ito stochastic integral equation

$$
x_{i}(t)=x_{i}(0)+\int_{0}^{t} d s A_{i}\left[x_{i}(s), s\right]+\int_{0}^{t} d W^{j}(s) B_{i j}\left[x_{i}(s), s\right]
$$

in which $x_{i}(t)$ is the variable of interest, the vector $A_{i}[x(t), t]$ denotes deterministic drift, the matrix $B_{i j}[x(t), t]$ represents continuous stochastic diffusion fluctuations, and $W^{j}(t)$ is an $N$-variable Wiener process (i.e., generalized Brownian motion), and $d W^{j}(t)=W^{j}(t+d t)-W^{j}(t)$. 
The Markov assumption is formulated in terms of the conditional probabilities $P\left(x_{i}, t_{i}\right)$ : if the times $t_{i}$ increase from right to left, the conditional probability is determined entirely by the knowledge of the most recent condition. Markov process is generated by a set of conditional probabilities whose probability-density function $P\left(x^{\prime}, t^{\prime} \mid x^{\prime \prime}, t^{\prime \prime}\right)$ obeys the general Chapman-Kolmogorov integro-differential equation [4]

$$
\begin{aligned}
\partial_{t} P\left(x^{\prime}, t^{\prime} \mid x^{\prime \prime}, t^{\prime \prime}\right)= & -\sum_{i} \frac{\partial}{\partial x^{i}}\left\{A_{i}[x(t), t] P\left(x^{\prime}, t^{\prime} \mid x^{\prime \prime}, t^{\prime \prime}\right)\right\} \\
+ & \frac{1}{2} \sum_{i j} \frac{\partial^{2}}{\partial x^{i} \partial x^{j}}\left\{B_{i j}[x(t), t] P\left(x^{\prime}, t^{\prime} \mid x^{\prime \prime}, t^{\prime \prime}\right)\right\} \\
& +\int d x\left\{W\left(x^{\prime} \mid x^{\prime \prime}, t\right) P\left(x^{\prime}, t^{\prime} \mid x^{\prime \prime}, t^{\prime \prime}\right)\right. \\
& \left.-W\left(x^{\prime \prime} \mid x^{\prime}, t\right) P\left(x^{\prime}, t^{\prime} \mid x^{\prime \prime}, t^{\prime \prime}\right)\right\}
\end{aligned}
$$

including: deterministic drift, diffusion fluctuations, and discontinuous jumps (given, respectively, in the first, second, and third rows).

In terms of the Markov stochastic process (2.12), (2.13), and (2.14), we can interpret our deterministic $\delta$-Hamiltonian HB-system (2.5), (2.6), and (2.7) as deterministic drift corresponding to the Liouville equation. Thus, we can naturally (in the sense of Langevin) add the covariant vector $\sigma_{i}(t)$ of stochastic forces (diffusion fluctuations and discontinuous-Master jumps) $\sigma_{i}(t)=B_{i j}\left[q^{i}(t), t\right] d W^{j}(t)$-to the canonical force equation. In this way we obtain stochastic $\sigma$-Hamiltonian HB-system, a stochastic transformation Stoch [Can] of the canonical functor Can,

$$
\begin{aligned}
d q^{i} & =\left(\frac{\partial H}{\partial p_{i}}+\frac{\partial R}{\partial p_{i}}\right) d t \\
d p_{i} & =\left(F_{i}-\frac{\partial H}{\partial q^{i}}+\frac{\partial R}{\partial q^{i}}\right) d t+\sigma_{i}(t), \\
\sigma_{i}(t) & =B_{i j}\left[q^{i}(t), t\right] d W^{j}(t), \\
q^{i}(0) & =q_{0}^{i}, \quad p_{i}(0)=p_{i}^{0} .
\end{aligned}
$$

In our case of symmetrical 3D load-lifting, the velocity and force $\sigma$-Hamiltonian HB-equations (2.8) and (2.9) become

$$
\begin{aligned}
d q^{i}= & \left(p_{i}\left\{\left[J_{i}\right]^{-1}+\left[m_{i}\left(\sum_{j=1}^{i} L_{j} \cos q^{j}\right)^{2}\right]^{-1}\right\}+\frac{\partial R}{\partial p_{i}}\right) d t, \\
d p_{i}= & B_{i j}\left[q^{i}(t), t\right] d W^{j}(t) \\
& +\left(F_{i}-g \sum_{j=i}^{10-i} L_{j} m_{j} \sin q^{j}-\sum_{j=i}^{10-i} L_{j} \sin q^{j} p_{i} p_{j}\left[m_{i}\left(\sum_{k=1}^{i} L_{k} \cos q^{k}\right)^{3}\right]^{-1}+\frac{\partial R}{\partial q^{i}}\right) d t .
\end{aligned}
$$


Through stochastic $\sigma$-Hamiltonian HB-system (2.15), the deterministic $\delta$ Hamiltonian phase-flow $\phi_{\delta_{t}}$ (2.10), extends into stochastic $\sigma$ Hamiltonian phase-flow

$$
\begin{gathered}
\phi_{\sigma_{t}}: G_{1} \times I^{*} Q^{N} \longrightarrow I^{*} Q^{N},(p(0), q(0)) \longmapsto(p(t), q(t)), \\
\left(\phi_{\sigma_{t}} \circ \phi_{\sigma_{s}}=\phi_{\sigma_{t+s}}, \phi_{\sigma_{0}}=\text { identity }\right),
\end{gathered}
$$

where $I^{*} Q^{N}$ denotes Ito quadratic cotangent bundle (see [11]) of HB-configuration manifold $Q^{N}$. Ito quadratic cotangent bundle $I^{*} Q^{N}$ is defined as a Whitney sum

$$
I^{*} Q^{N}=T^{*} Q^{N} \oplus S Q^{N}
$$

where $S Q^{N}$ corresponds to stochastic tensor bundle whose elements are 2nd-order tensor fields composed of continual diffusion fluctuations and discontinuous jumps at every point of the manifold $Q^{N}$. On $I^{*} Q^{N}$ is defined a non-degenerate, stochastic two-form $\alpha$ which is closed, that is, $d \alpha=0$, and exact, that is, $\alpha=d \beta$, where one-form $\beta$ represents a section $\beta: Q^{N} \rightarrow I^{*} Q^{N}$ of the Ito bundle $I^{*} Q^{N}$.

The quadratic character of Ito stochastic fiber-bundles corresponds to the second term (trace of the 2nd-order tensor) of associate stochastic Taylor expansion (see [11]).

Besides the $\sigma$-Hamiltonian phase-flow $\phi_{\sigma_{t}}(2.17)$, including $N$ individual randomphase trajectories, we can also define (see $[4,16])$ a mean $\langle\sigma\rangle$-Hamiltonian flow $\langle\phi\rangle_{\sigma_{t}}$

$$
\begin{gathered}
\langle\phi\rangle_{\sigma_{t}}: G_{1} \times I^{*} Q^{N} \longrightarrow I^{*} Q^{N},(\langle p(0)\rangle,\langle q(0)\rangle) \longmapsto(\langle p(t)\rangle,\langle q(t)\rangle), \\
\left(\langle\phi\rangle_{\sigma_{t}} \circ\langle\phi\rangle_{\sigma_{s}}=\langle\phi\rangle_{\sigma_{t+s}},\langle\phi\rangle_{\sigma_{0}}=\text { identity }\right),
\end{gathered}
$$

which gives a sort of stochastic approximation for trajectory of the centre of mass in $\mathrm{HB}$, usually (in biomechanical literature) taken to be the lumbo-sacral SO(3)-joint located at the lower third of the spine (see Figure 2.1).

The necessary conditions for existence of a unique nonanticipating solution of $\sigma$ Hamiltonian HB-system in a fixed time interval are Lipschitz condition and growth condition $[11,16]$. For constructing an approximate solution a simple iterative CauchyEuler procedure could be used to calculate $\left(q_{k+1}^{i}, p_{i}^{k+1}\right)$ from the knowledge of $\left(q_{k}^{i}, p_{i}^{k}\right)$ on the mesh of time points $t^{k}, k=1, \ldots, s$, by adding discrete $\delta$-Hamiltonian driftterms $A^{i}\left(q_{k}^{i}\right) \Delta t^{k}$ and $A_{i}\left(p_{i}^{k}\right) \Delta t^{k}$, as well as a stochastic term $B_{i j}\left(q_{i}^{k}, t^{k}\right) \Delta W_{k}^{j}$.

\section{Topology invariants of $\mathrm{HB}$}

3.1. Cohomology groups of HB. If $\mathscr{C}=H^{\bullet} M$ represents the Abelian category of cochains [1, 2] on the momentum phase-space manifold $T^{*} Q^{N}$, we have the category $\mathscr{S}^{\bullet}\left(H^{\bullet} M\right)$ of generalized cochain complexes $A^{\bullet}$ in $\mathscr{H}^{\bullet} M$ and if $A_{n}^{\prime}=0$ for $n<0$, we have a subcategory $\mathscr{S}_{\mathrm{DR}}^{\bullet}\left(H^{\bullet} M\right)$ of de Rham differential complexes in $\mathscr{S}^{\bullet}\left(H^{\bullet} M\right)$

$$
\begin{aligned}
A_{\mathrm{DR}}^{\cdot}: 0 & \longrightarrow \Omega^{0}\left(T^{*} Q^{N}\right) \stackrel{d}{\longrightarrow} \Omega^{1}\left(T^{*} Q^{N}\right) \\
& \stackrel{d}{\longrightarrow} \Omega^{2}\left(T^{*} Q^{N}\right) \stackrel{d}{\longrightarrow} \cdots \stackrel{d}{\longrightarrow} \Omega^{N}\left(T^{*} Q^{N}\right) \stackrel{d}{\longrightarrow} \cdots,
\end{aligned}
$$

where $A_{N}^{\prime}=\Omega^{N}\left(T^{*} Q^{N}\right)$ is the vector space of all $N$-forms on $T^{*} Q^{N}$ over $\mathbb{R}$. 
Let $Z^{N}\left(T^{*} Q^{N}\right)=\operatorname{Ker}(d)$ and $B^{N}\left(T^{*} Q^{N}\right)=\operatorname{Im}(d)$ denote, respectively, the real vector spaces of cocycles and coboundaries of degree $N$. Since $d_{N+1} d_{N}=d^{2}=0$, it follows that $B^{N}\left(T^{*} Q^{N}\right) \subset Z^{N}\left(T^{*} Q^{N}\right)$. The quotient vector space

$$
H_{\mathrm{DR}}^{N}\left(T^{*} Q^{N}\right)=\operatorname{Ker}(d) / \operatorname{Im}(d)=Z^{N}\left(T^{*} Q^{N}\right) / B^{N}\left(T^{*} Q^{N}\right)
$$

is referred to as the de Rham cohomology group (vector space) of HB on $T^{*} Q^{N}$. The elements of $H_{\mathrm{DR}}^{N}\left(T^{*} Q^{N}\right)$ are equivalence sets of cocycles. Two cocycles $\omega_{1}$ and $\omega_{2}$ are cohomologous, or belong to the same equivalence set (written $\omega_{1} \sim \omega_{2}$ ) if and only if they differ by a coboundary $\omega_{1}-\omega_{2}=d \theta$. Any form $\omega_{H} \in \Omega^{N}\left(T^{*} Q^{N}\right)$ has a de Rham cohomology class $\left[\omega_{H}\right] \in H_{\mathrm{DR}}^{N}\left(T^{*} Q^{N}\right)$.

Hamiltonian symplectic form $\omega_{H}=d p_{i} \wedge d q_{i}$ on $T^{*} Q^{N}$ is by definition both a closed two-form or two-cocycle and an exact two-form or two-coboundary. Therefore, the two-dimensional de Rham cohomology group of HB is defined as a quotient vector space

$$
H_{\mathrm{DR}}^{2}\left(T^{*} Q^{N}\right)=Z^{2}\left(T^{*} Q^{N}\right) / B^{2}\left(T^{*} Q^{N}\right)
$$

As $T^{*} Q^{N}$ is a compact Hamiltonian symplectic manifold of dimension $2 N$, it follows that $\omega_{H}^{N}$ is a volume element on $T^{*} Q^{N}$, and the $2 N$-dimensional de Rham cohomology class $\left[\omega_{H}^{N}\right] \in H_{\mathrm{DR}}^{2 N}\left(T^{*} Q^{N}\right)$ is nonzero. Since $\left[\omega_{H}^{N}\right]=\left[\omega_{H}\right]^{N}$, then $\left[\omega_{H}\right] \in H_{\mathrm{DR}}^{2}\left(T^{*} Q^{N}\right)$ and all of its powers up to the $N$ th must be zero as well. The existence of such an element is a necessary condition for $T^{*} Q^{N}$ to admit a Hamiltonian symplectic structure $\omega_{H}$.

A de Rham complex $A_{\mathrm{DR}}^{\cdot}$ on $T^{*} Q^{N}$ can be considered as a system of second-order differential equations $d^{2} \theta_{H}=0, \theta_{H} \in \Omega^{N}\left(T^{*} Q^{N}\right)$ having a solution represented by $Z^{N}\left(T^{*} Q^{N}\right)$. In local coordinates $q^{i}, p_{i} \in U_{p}\left(U_{p}\right.$ open in $\left.T^{*} Q^{N}\right)$, we have $d^{2} \theta_{H}=$ $d^{2}\left(p_{i} d q^{i}\right)=d\left(d p_{i} \wedge d q^{i}\right)=0$.

3.2. Homology groups of HB. If $\mathscr{C}=H . M$ represents an Abelian category of chains $[1,2]$ on $T^{*} Q^{N}$, we have a category $\mathscr{Y}_{\bullet}\left(H_{\bullet} M\right)$ of generalized chain complexes $\mathscr{A}_{\bullet}$ in $\mathcal{H}_{\bullet} M$, and if $A=0$ for $n<0$, we have a sub-category $\mathscr{Y}_{\bullet}^{C}\left(H_{\bullet} M\right)$ of chain complexes in H.M

$$
A_{.}: 0 \longleftarrow C^{0}\left(T^{*} Q^{N}\right) \stackrel{\partial}{\rightleftarrows} C^{1}\left(T^{*} Q^{N}\right) \stackrel{\partial}{\longleftarrow} C^{2}\left(T^{*} Q^{N}\right) \stackrel{\partial}{\longleftarrow} \ldots \stackrel{\partial}{\longleftarrow} C^{n}\left(T^{*} Q^{N}\right) \stackrel{\partial}{\longleftarrow} \cdots .
$$

Here $A_{N}=C^{N}\left(T^{*} Q^{N}\right)$ is the vector space of all finite chains $C$ on $T^{*} Q^{N}$ over $\mathbb{R}$, and $\partial_{N}=\partial: C^{N+1}\left(T^{*} Q^{N}\right) \rightarrow C^{N}\left(T^{*} Q^{N}\right)$. A finite chain $C$ such that $\partial C=0$ is an $N$-cycle. A finite chain $C$ such that $C=\partial B$ is an $N$-boundary. Let $Z_{N}\left(T^{*} Q^{N}\right)=\operatorname{Ker}(\partial)$ and $B_{N}\left(T^{*} Q^{N}\right)=\operatorname{Im}(\partial)$ denote, respectively, real vector spaces of cycles and boundaries of degree $N$. Since $\partial_{N-1} \partial_{N}=\partial^{2}=0$, then $B_{N}\left(T^{*} Q^{N}\right) \subset Z_{N}\left(T^{*} Q^{N}\right)$. The quotient vector space

$$
H_{N}^{C}\left(T^{*} Q^{N}\right)=Z_{N}\left(T^{*} Q^{N}\right) / B_{N}\left(T^{*} Q^{N}\right)
$$


represents an $N$-dimensional homology group (vector space) of humanoid dynamics. The elements of $H_{N}^{C}\left(T^{*} Q^{N}\right)$ are equivalence sets of cycles. Two cycles $C_{1}$ and $C_{2}$ are homologous, or belong to the same equivalence set (written $C_{1} \sim C_{2}$ ) if and only if they differ by a boundary $C_{1}-C_{2}=\partial B$. The homology class of a finite chain $C \in C^{N}\left(T^{*} Q^{N}\right)$ is $[C] \in H_{N}^{C}\left(T^{*} Q^{N}\right)$.

Similar (co)homology analysis could be also performed on the stochastic Ito quadratic cotangent bundle $I^{*} Q^{N}$ of HB.

3.3. Reduced configuration manifold: Betti numbers and Euler-Poincaré characteristic. The HB-configuration manifold $Q^{N}$ (Figure 2.1) can be, for the sake of the brain-like motor control [5], reduced to $N$-torus $T^{N}$, in three steps, as follows.

First, a single three-axial SO(3)-joint can be reduced to the direct product of three uniaxial SO(2)-joints, in the sense that three hinge joints can produce any orientation in space, just as a ball-joint can. Algebraically, this means reduction of each of the three $\mathrm{SO}$ (3) rotation matrices to the corresponding $\mathrm{SO}(2)$ rotation matrices, by deleting the rows and columns with zeros and 1.

In this way, we can set the reduction equivalence relation $\mathrm{SO}(3) \gtrsim \mathrm{SO}(2) \times \mathrm{SO}(2) \times$ $\mathrm{SO}(2)$.

Second, we have a homeomorphism: $\mathrm{SO}(2) \sim S^{1}$, where $S^{1}$ denotes the constrained unit circle in the complex plane, which is an Abelian Lie group.

Third, let $I^{N}$ be the unit cube $[0,1]^{N}$ in $\mathbb{R}^{N}$ and “ " an equivalence relation on $\mathbb{R}^{N}$ obtained by gluing together the opposite sides of $I^{N}$, preserving their orientation. The manifold of humanoid configurations depicted in Figure 2.1 can be represented as a quotient space of $\mathbb{R}^{N}$ by the space of the integral lattice points in $\mathbb{R}^{N}$, that is a constrained $N$-dimensional torus $T^{N}$ :

$$
\mathbb{R}^{N} / Z^{N}=I^{N} / \sim \cong \prod_{i=1}^{N} S_{i}^{1} \equiv\left\{\left(q^{i}, i=1, \ldots, N\right): \bmod (2 \pi)\right\}=T^{N}
$$

Since $S^{1}$ is an Abelian Lie group, its $N$-fold direct product $T^{N}$ is also an Abelian Lie group, the toral group, of all nondegenerate diagonal $N \times N$ matrices. As a Lie group, the HB-configuration space $Q^{N} \equiv T^{N}$ has a natural Banach manifold structure with local internal coordinates $q^{i} \in U, U$ being an open set (chart) in $T^{N}$.

Conversely by ungluing the configuration space, we obtain the primary unit cube. Let “ * " denote an equivalent decomposition or ungluing relation. By the Tychonoff product topology theorem, for every such quotient space there exists a selector such that their quotient models are homeomorphic, that is, $T^{N} / \sim^{*} \approx A^{N} / \sim^{*}$. Therefore, $I^{N}$ represents a selector for the configuration torus $T^{N}$ and can be used as an $N$ directional command-space for the topological control of humanoid motion. Any subset of degrees of freedom on the configuration torus $T^{N}$ (3.6) representing the joints included in humanoid motion has its simple, rectangular image in the command space-selector $I^{N}$. Operationally, this resembles what the brain motor controller, actually performs on the highest level of human motor control (see [14, 15]).

Now, the Betti numbers for the configuration torus $T^{N}$ (3.6) follow from (3.2) and (3.5), using the homotopy axiom for de Rham cohomologies, as well as the de Rham 
theorem

$$
\begin{aligned}
& b^{0}=1, \\
& b^{1}=N, \ldots, b^{p}=\left(\begin{array}{l}
N \\
p
\end{array}\right), \ldots, b^{N-1}=N, \\
& b^{N}=1 \quad(0 \leq p \leq N) .
\end{aligned}
$$

Therefore, $b^{N}=b_{N}$ are given by (3.7) for both $T^{N}$ and $T^{*} T^{N}$, defining also their EulerPoincaré characteristic as [1]

$$
\chi\left(T^{N}, T^{*} T^{N}\right)=\sum_{p=1}^{N}(-1)^{p} b_{p} .
$$

\section{REFERENCES}

[1] Y. Choquet-Bruhat, C. DeWitt-Morette, and M. Dillard-Bleick, Analysis, Manifolds and Physics, 2nd ed., North-Holland Publishing, Amsterdam, 1982.

[2] C. T. J. Dodson and P. E. Parker, A User's Guide to Algebraic Topology, Mathematics and Its Applications, vol. 387, Kluwer Academic Publishers, Dordrecht, 1997.

[3] M. Dorigo and U. Schnepf, Genetics-based machine learning and behavior-based robotics: a new synthesis, IEEE Trans. Systems Man Cybernet. 23a (1993), 141-154.

[4] C. W. Gardiner, Handbook of Stochastic Methods. For Physics, Chemistry and the Natural Sciences, 2nd ed., Springer Series in Synergetics, vol. 13, Springer-Verlag, Berlin, 1985.

[5] J. C. Houk, J. T. Buckingham, and A. G. Barto, Models of the cerebellum and motor learning, Behav. Brain Sci. 19 (1996), 368-383.

[6] Y. Hurmuzlu, Dynamics of bipedal gait, J. Appl. Mech. 60 (1993), 331-343.

[7] V. Ivancevic, Introduction to Biomechanical Systems: Modeling, Control and Learning, Scientific Book, Belgrade, 1991.

[8] V. Ivancevic and M. Snoswell, Fuzzy stochastic functor machine for general humanoid robot dynamics, IEEE Trans. Systems Man Cybernet. 31B (2001), no. 3, 319-330.

[9] H. Ko and N. Badler, Animating human locomotion with inverse dynamics, IEEE Comput. Graph. \& Appl. 16 (1996), 50-59.

[10] S. MacLane, Categories for the Working Mathematician, Graduate Texts in Mathematics, vol. 5, Springer-Verlag, New York, 1971.

[11] P. A. Mayer, A Differential Geometric Formalism for the Ito Calculus, Springer, New York, 1981.

[12] C. Pribe, S. Grossberg, and M. A. Cohen, Neural control of interlimb oscillations. II, biped and quadruped gaits and bifurcations, Biol. Cybernet. 77 (1997), 141-152.

[13] P. Sardain, M. Rostami, and G. Bessonnet, An anthropomorphic biped robot: dynamic concepts and technological design, IEEE Trans. Systems Man Cybernet. 28a (1999), no. 6, 823-838.

[14] S. Schaal, Is imitation learning the route to humanoid robots? Trends in Cogn. Sci. 3 (1999), no. $6,233-242$.

[15] S. Schaal and C. G. Atkeson, Constructive incremental learning from only local information, Neural Computation. 10 (1998), no. 8, 2047-2084.

[16] H. Schurz, Moment attractivity, stability and contractivity exponents of stochastic dynamical systems, Discrete Contin. Dynam. Systems 7 (2001), no. 3, 487-515.

[17] C. L. Shih, W. Gruver, and T. Lee, Inverse kinematics and inverse dynamics for control of a biped walking machine, Journal of Robotic Systems 10 (1993), no. 5, 531-555.

[18] M. Vukobratovic, Legged Locomotion Robots and Anthropomorphic Mechanisms, Mihailo Pupin, Belgrade, 1975. 
[19] M. Vukobratovic, B. Borovac, D. Surla, and D. Stokic, Biped Locomotion: Dynamics, Stability, Control, and Applications, Springer-Verlag, Berlin, 1990.

[20] T. Yoshikawa, Analysis and control of robot manipulators with redundancy, Robotics Research (M. Brady and R. Paul, eds.), MIT Press, Massachusetts, 1984, pp. 735-747.

Vladimir IVAncevic: Human Factors, Land Operation Division, Defence Science and TeChNology Organization, P.O. Box 1500, Edinburgh 5111, SA, Australia

E-mail address: V1adimi r. Ivancevic@dsto.defence.gov.au 


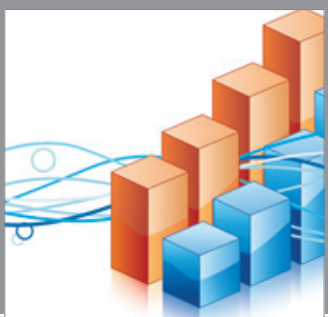

Advances in

Operations Research

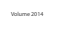

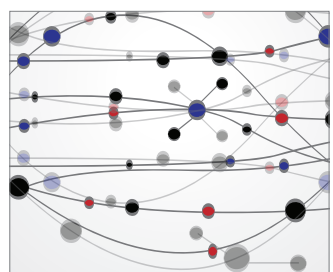

\section{The Scientific} World Journal
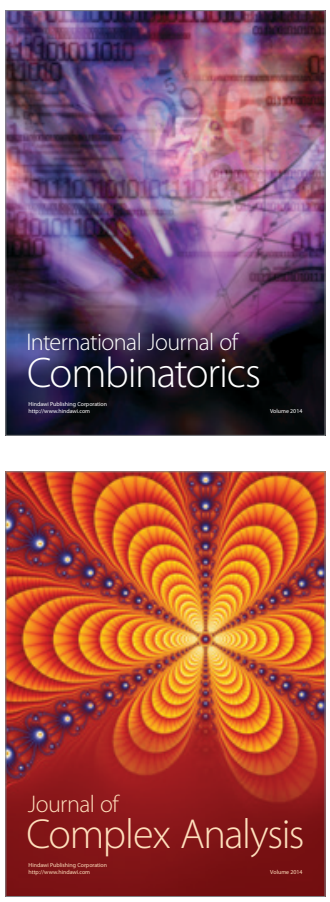

International Journal of

Mathematics and

Mathematical

Sciences
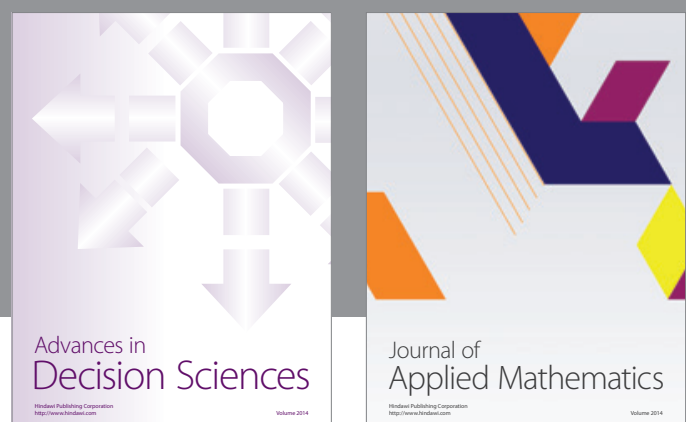

Journal of

Applied Mathematics
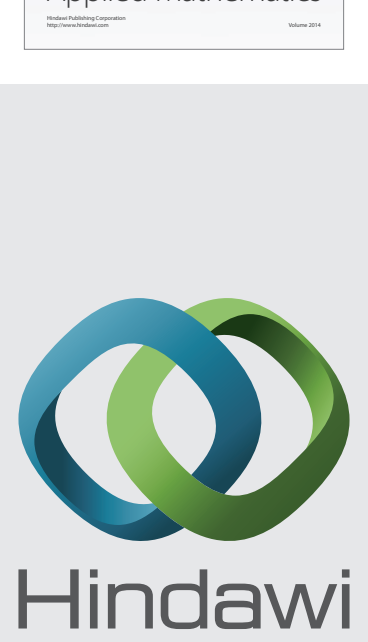

Submit your manuscripts at http://www.hindawi.com
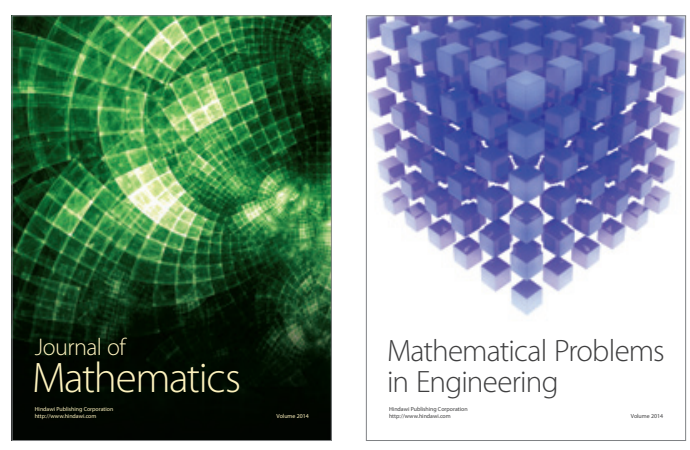

Mathematical Problems in Engineering
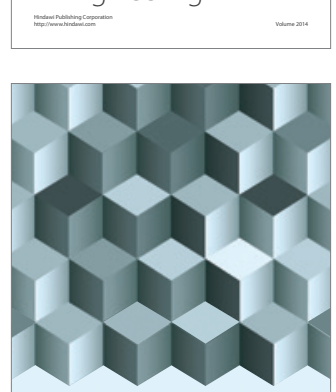

Journal of

Function Spaces
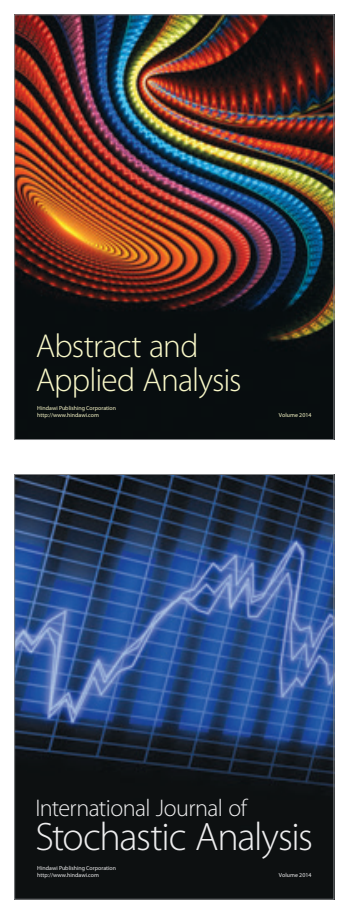

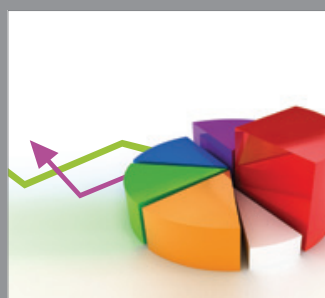

ournal of

Probability and Statistics

Promensencen
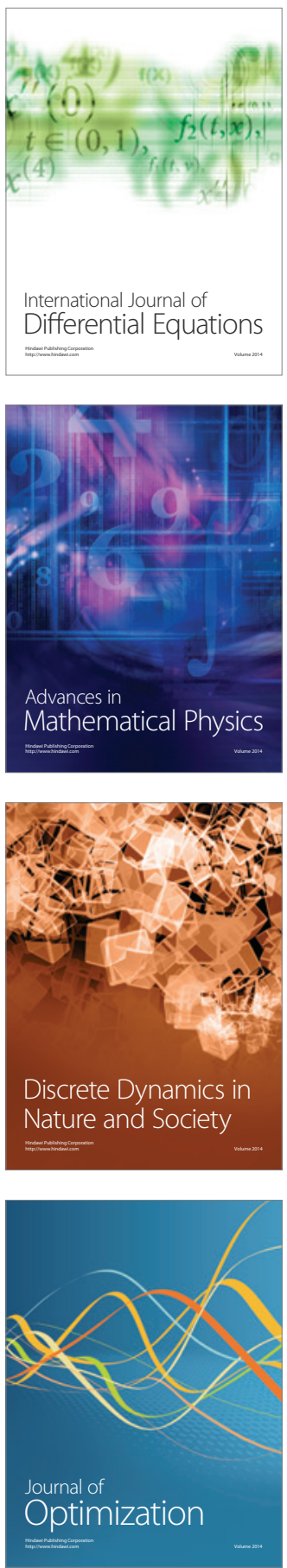